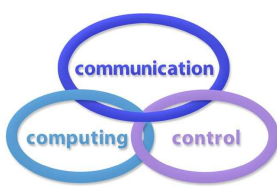

\title{
Sensor Coverage Strategy in Underwater Wireless Sensor Networks
}

\author{
L. Yao, X. Du
}

\author{
Lu Yao \\ Computer Department \\ Qinghai Normal University, Xining 810008, China \\ 401516575@qq.com
}

\section{Xiujuan Du*}

1. Computer Department

Qinghai Normal University, Xining 810008, China

2.Academy of Plateau Science and Sustainability

Xining 810008, China

*Corresponding author: dxj@qhnu.edu.cn

\begin{abstract}
This paper mainly describes studies hydrophone placement strategy in a complex underwater environment model to compute a set of "good" locations where data sampling will be most effective. Throughout this paper it is assumed that a $3-D$ underwater topographic map of a workspace is given as input.Since the negative gradient direction is the fastest descent direction, we fit a complex underwater terrain to a differentiable function and find the minimum value of the function to determine the low-lying area of the underwater terrain. The hydrophone placement strategy relies on gradient direction algorithm that solves a problem of maximize underwater coverage: Find the maximize coverage set of hydrophone inside a $3-D$ workspace. After finding the maximize underwater coverage set, to better take into account the optimal solution to the problem of data sampling, the finite VC-dimension algorithm computes a set of hydrophone that satisfies hydroacoustic signal energy loss constraints. We use the principle of the maximize splitting subset of the coverage set and the "dual" set of the coverage covering set, so as to find the hitting set, and finally find the suboptimal set (i.e., the sensor suboptimal coverage set).Compared with the random deployment algorithm, although the computed set of hydrophone is not guaranteed to have minimum size, the algorithm does compute with high network coverage quality.

Keywords: Underwater Wireless Sensor Networks(UWSNs), Finite VC-dimension, Gradient direction, Sensor deployment.
\end{abstract}

\section{Introduction}

In recent years, with the rapid development of underwater sensor network research and applications and the improvement in hardware technology, sensor nodes have the ability to collect various resources and information underwater [5],[19],[15]. Sensor nodes suitable for underwater environments can be cooperatively combined. An underwater wireless sensor networks (UWSNs) is formed via acoustic 
communication. Regardless of the application of the UWSNs, the deployment problem of the sensor node is first solved because it directly affects the accuracy and comprehensiveness of the monitoring results[11]. A reasonable and effective sensor deployment solution can greatly reduce the network construction time, quickly cover the underwater range, and extend the network life[28].

Precise UWSNs sensor node deployment is the foundation of building a sensor network, and it is a prominent issue of research. A good node deployment strategy has an important impact on network performance and network survivability [3]. The underwater sensor node deployment problem is the organic combination of the front-end information collection and back-end data processing of the UWSNs system and is the fundamental challenge of the sensor network. At the application site, node deployment needs to be performed through appropriate scheduling algorithms. At present, the research on underwater network coverage mostly adopts an ideal coverage environment model [8],[25]. The perceived area of the node is considered to be a shape-formed prototype area, and the perceived radius of the node is the same in all directions; the effects of irregular shapes in the area of sensor coverage are not considered. Precise sensor coverage in a relatively harsh environment, such as the detection and sampling of underwater objects, can affect underwater warfare and early warning and water pollution prevention systems as well as other applications [24],[30]. Although the node layout can adopt the dense sensor random deployment strategy (which carries high labor and material costs), sensor signals are susceptible to environmental and material obstructions. The node perception range is rendered irregular, and monitoring blind zones are prone to occur. Based on this perspective, this paper presents a scheduling algorithm for randomly deploying sensors in complex underwater models.

The complex underwater environment is modeled by measuring terrain data. Terrains, basins, continental shelves, continental slopes, isolated islands, and mid-ocean ridges in underwater terrain are already included in Fig.1(a)(considering computational complexity, $3-D$ models have been scaled down).At present, sonar technology has been used to better measure the seabed topographic map. To facilitate calculation and demonstration, the cross-sectional view required by this model, such as the $2-D$ workspace map see [14], is shown in Fig.1(b). The model takes the underwater terrain into account, thus verifying the reasonability and effectiveness of the scheduling algorithm.

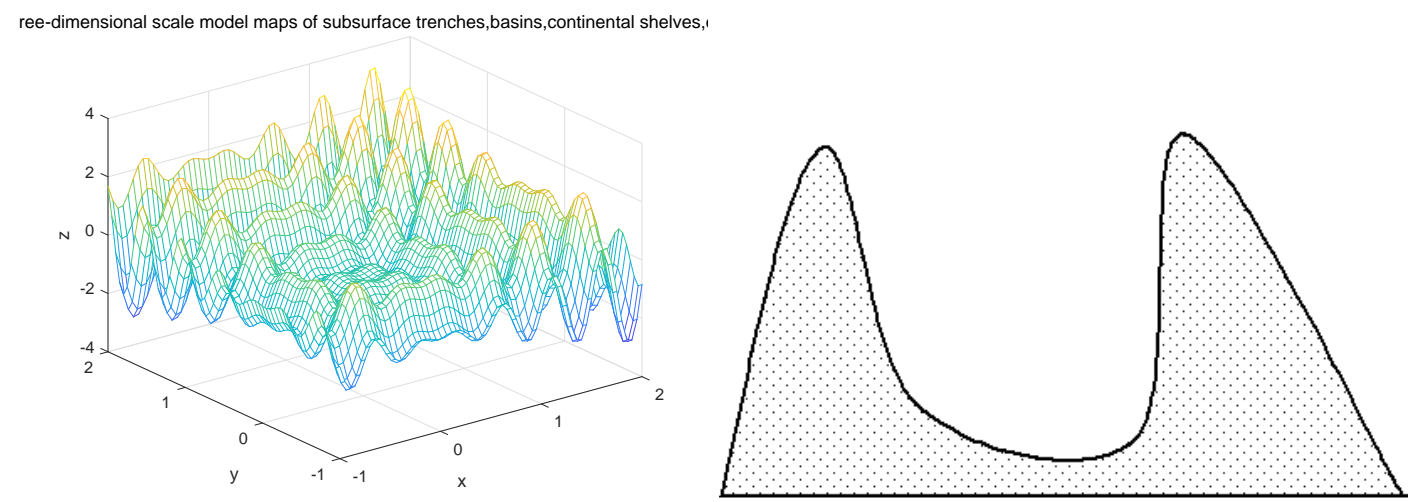

(a) Three-dimensional scale model maps of sub- (b) Cross-sectional pattern containing the trench. surface trenches, basins, continental shelves, etc.

Figure 1: Underwater topographic map and cross section.

The basic principle of using a scheduling algorithm to complete underwater environment deployment is that the greater the probability $\mathcal{P}$ of effective sensor deployment, the better the algorithm $\mathcal{A}$. Here, $\mathcal{P}\left(\mathcal{S}_{i} \mid \mathbb{R}^{3}, \mathcal{A}\right)$ represents the probability of effective deployment by the $\mathcal{A}$ algorithm in the case of $\mathbb{R}^{3}, \mathbb{R}^{3}$ represents complex underwater terrain, $\mathcal{S}$ is the sensor node, and $\mathcal{A}$ represents the scheduling algorithm. The $\mathcal{A}$ algorithm consists of a two-part algorithm; one part is able to achieve maximum range coverage in the $3-D$ environment(See Algorithm1), and the other part optimizes the hydrophone node set under the maximum range coverage condition(See Algorithm2). In Section 3, the iterative algorithm is used to obtain the gradient vector direction of each part of either the trench or mid-ocean ridge. The hydrophone adjusts the sinking position according to the gradient direction symbol to ensure the maximum underwater area. In Section 4, the finite VC-dimension algorithm 
is used to obtain an approximate optimal hydrophone node coverage set, thereby reducing redundant hydrophone nodes.

\section{Related work}

In recent years, many researchers have begun to develop algorithms for underwater sensor deployment. Different deployment algorithms have been proposed according to different application scenarios and requirements. The common feature of these algorithms is that the sensor nodes use either static deployment algorithms or dynamic random deployment algorithms.

\subsection{Creation of static deployment algorithm}

Static deployment is widely used in underwater sensor networks. When either the underwater environment is calm or the network state is relatively stable, the hydrophone nodes are generally calculated and deployed at predetermined locations according to the application requirements. For the problem of node deployment in underwater $3-D$ environments, He, Ming et al. proposed an adaptive algorithm for $2-D$ to $3-D$ space deployment strategies. See [23], a series of rules for effectively constructing $3-D$ network topologies are proposed to resolve node deployment problems and planning in $3-D$ space. The problem of $3-D$ space node deployment is approached by conversion to a $2-D$ problem. In [26], the author obtains the number of active nodes required for basic network coverage and connectivity under the grid deployment model. However, the algorithm is applicable only to $2-D$ environments and cannot meet the application requirements of a $3-D$ environment.

See [20], the use of multiple connected underwater sensors was suggested. Combined with the deployment characteristics of $3-D$ space, a deployment algorithm based on network connectivity performance was proposed.Regarding the dense deployment of sensor nodes, a deployment method with nodes having the longest lifetime is recommended to optimize the coverage set.

Static deployment algorithms are typically used in applications that either take place in uncomplicated environments or have a relatively stable network state. Hydrophone nodes are placed at locations that are determined and calculated based on application requirements [27]. In this case, the hydrophone node deployment problem is usually abstracted into a linear programming problem, and the proposed solution is optimized for either network performance or cost. However, in a natural underwater acoustic environment, the location of hydrophone node deployment is often undetermined due to either the uncertainty of the underwater environment or the undetectable nature of the monitoring environment. In this case, the dynamic feature of the underwater sensor node is applied, although the coverage performance of the sensor cannot be guaranteed.

\subsection{Dynamic random deployment algorithm}

In [18], the nodes have mobility. After being deployed in the target area, each node uses its repulsive force to move in the opposite direction of the neighboring nodes until the repulsive forces from the various directions are balanced by the nodes. This method reduces the overlap area between nodes. The VFA algorithm proposed by Zou and Chakrabarty is also based on the addition of node mobility functional modules [31]. The VFA first performs the mobility simulation. After determining the locations of the mobile nodes, all of the nodes move to the specified positions at one time. Algorithm deployment is highly efficient, and the algorithm complexity varies according to the number of nodes and the size of the target area. The algorithm complexity of deploying $k$ nodes in a given area divided into $n \times m$ grids is $O(n m k)$.

See [7], a new sensor network structure is introduced, and an underwater sensor network node deployment algorithm based on a random surface configuration is proposed. During the initial configuration of the network, several nodes are randomly deployed on the horizontal plane, and then the depth of neighboring nodes in the spatial adjustment area is arranged according to each node so that the underwater $3-D$ space is sufficiently covered.Arvind et al. proposed an energy modelbased method designed to extend the life of the network by balancing motion and energy loss in the network,experiments have proved the effectiveness of the method[12]. 


\section{$3 \quad 3-D$ sensor space coverage problem}

The $3-D$ underwater environment hydrophone deployment problem is a leading issue in sensor deployment research [17]. Although many scholars have developed high-coverage and low-complexity solutions, many algorithms are theoretical because they are based on an ideal environment; the practicality of these algorithms in complex underwater terrain environments remains to be confirmed [21]. In this paper, we roughly abstract the actual terrain, such as underwater trenches and basins, into curved surfaces from the actual underwater terrain data provided(in the actual calculation process, the deployment position of the sensor does not need to be accurately calculated, and this does not affect the calculation result). The direction of bumps in the complex terrain is calculated by the gradient direction method (called the trench, terrain such as the basin is concave terrain, and the topography of the mid-ocean ridge is convex terrain)[10],[29]. According to the direction of the bump, the range in which the hydrophone node needs to be deployed with high probability can be calculated.

Problem 1. (Complex terrain hydrophone node coverage):For a given range of $3-D$ complex environments $\mathbb{R}^{3}$, effectively calculate the maximize underwater coverage set of hydrophone $\mathcal{S}=$ $\left\{s_{1}, s_{2}, s_{3}, \ldots, s_{n}\right\} ;$ thus, in the environment $\mathbb{R}^{3}$, each complex terrain is guaranteed to have hydrophone node coverage.

Definition 2. Assume that the slope, including the slope and the approximately smooth surface, is referred to as a continuous surfacefor which $\mathcal{M} \in \mathbb{R}^{3}, \mathcal{M}=\left\{m_{1}, m_{2}, \ldots, m_{n}\right\}$, and $m_{i}(1<i<n)$ divides the surface $\mathcal{M}$ into a smaller surface. $h(m)$ is a smooth surface fitted by the section $m_{i}$ in $3-D$ space. Then, Loss function $J(\theta)$ can be expressed as in Equation (1) :

$$
J(\theta)=\frac{1}{n} \sum_{i=1}^{n}\left[h_{\theta}\left(m_{i}\right)-m_{i}\right]^{2}
$$

where $\theta$ represents the gradient direction.

In order to minimize the value of $J(\theta)$, the partial derivative of $J(\theta)$ can be obtained from (1):

$$
\frac{\partial J(\theta)}{\partial \theta}=-\frac{1}{n^{2}} \sum_{i=1}^{n}\left[h_{\theta}\left(m_{i}\right)-m_{i}\right]^{2} \cdot m_{i}
$$

Since the hydrophone $\mathcal{S}$ is adjusted in the gradient direction, the parameter $\theta$ must be updated in the negative gradient direction:

$$
\theta \leftarrow \theta-\frac{1}{n^{2}} \sum_{i=1}^{n}\left[h_{\theta}\left(m_{i}\right)-m_{i}\right]^{2} \cdot m_{i}
$$

According to Equation (3), the topographic trend of irregular underwater terrain can be conveniently calculated. If $\theta$ is either a decreasing function or a negative gradient direction, then the surface area is concave terrain, which commonly causes blind spots for the hydrophone. When deploying a hydrophone, the position of the hydrophone should be adjusted to increase the probability that the collection area covers the blind spot,as shown in Fig.2(a).

Restrictions 3. A hydrophone, $s_{i} \in \mathcal{S}$, is effectively deployed in the designated area if $s_{i}$ meets the following conditions:

- Energy loss restrictions: In the $3-D$ underwater environment $\mathbb{R}^{3}$, the the acoustic signal propagation loss TL is mainly composed of two parts: expansion consumption and attenuation consumption.i.e.TL $=10 k \lg x+\alpha(f) x$. Where $x$ is the acoustic signal propagation distance, $k$ is the acoustic signal propagation factor (the general value is 1.5), $\alpha(f)$ is the absorption factor.

- Perceptual range restrictions: The sensor node $s_{i}$ in the $3-D$ space $\mathbb{R}^{3}$ has a coordinate of $G_{s_{i}}\left(x_{i}, y_{i}, z_{i}\right)$.The perceptual range is a ball $B_{i}$ centered on $G_{s_{i}}$ and radius $R_{s_{i}}$. At this time, in the space $\mathbb{R}^{3}$, the probability that any point $p(x, y, z)$ can be detected by node $s_{i}$ :

$$
P\left(s_{i}, p\right)=\left\{\begin{array}{l}
1, d\left(s_{i}, p\right) \leq R_{s_{i}} \\
0, d\left(s_{i}, p\right)>R_{s_{i}}
\end{array}\right.
$$



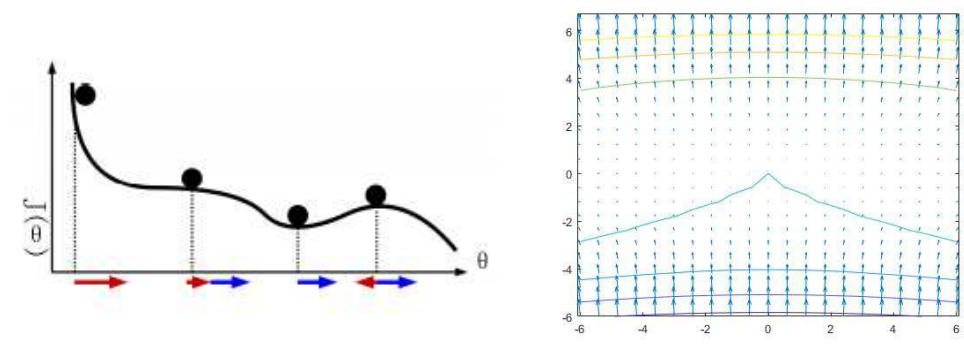

(a) In $3-D$ environment, the gra- (b) In the gradient pattern of the dient algorithm is used to get the $\theta$ depressed terrain in the $3-D$ graph, value, and the hydrophone adjusts the gradient vector in the range of the underwater depth according to 0 to -2 in the figure is the negative the $\theta$ value. direction, and the terrain can be determined as the depressed terrain.

Figure 2: The gradient direction algorithm calculates the $3-D$ underwater topographic map and the range of values of $\theta$.

where $d\left(s_{i}, p\right)=\sqrt{\left(x-x_{i}\right)^{2}+\left(y-y_{i}\right)^{2}+\left(z-z_{i}\right)^{2}}$ is European distance from $p$ to $s_{i}$.

- Terrain restrictions: $\mathcal{M}$ can be a smooth or nearly smooth terrain surface so that it can be divided into small enough $m_{i}$ to make $\mathcal{M} \approx h_{\theta}\left(m_{i}\right)$;

In a complex environment, the hydrophone is susceptible to obstacles, such as occlusion and there flection of sound off unsmooth objects; therefore, the above points need to be considered during the algorithm experiment. The above mentioned concentration restrictions also provide practical theoretical requirements for the feasibility of the algorithm. Submarine terrain that adhere to the characteristics of mathematical functions rarely occur in actual underwater environments. However, the various terrains on the seabed generally have a very large volume or area, and uneven terrain is abstracted into mathematical conformity by fitting methods. The surface of the model does not affect the results of the entire algorithm. Because the algorithm only needs to calculate the gradient vector of the fitted surface of the terrain and adjust the hydrophone to the negative region of the gradient vector, effective coverage in the current environment can be achieved.

Not all terrain can be covered by a hydrophone, such as in the case of Fig.3(a). In this case, underwater terrain data cannot be accurately obtained, which makes it impossible to calculate the direction of the gradient vector by Definition 2 because the terrain area has been completely separated from the outside world and the hydrophone cannot pass through the obstacle. The object communicates with an external hydrophone. Ultimately, the hydrophone task cannot be completed. We refer to Fig.3(b). as the sensing dead zone for a hydrophone node in a random deployment scenario[16]. Such blind areas mainly denote underwater concave terrain areas (including trenches, basins, etc.).

\subsection{Gradient direction algorithm based on complex terrain}

The gradient direction algorithm process is as follows Algorithm 1:

Step1:Because the negative gradient direction is the fastest falling direction of the function, the gradient direction of a certain point underwater can be calculated quickly. The gradient direction symbol of $\theta$ or $J(\theta)$ can be calculated by the given terrain data surface $\mathcal{M}$ to determine whether the area of the current data is concave terrain. Table 1 shows the coordinates of underwater terrain data example.By calculating the gradient of the coordinates of the point, the gradient direction of the specific region in Fig.2(b) is obtained.The gradient direction is calculated from this data, where "-" represents concave terrain.

Step2:Position the hydrophone node set $\mathcal{S}=\left\{s_{1}, s_{2}, s_{3}, \ldots, s_{n}\right\}$ to ensure that sufficient hydrophones are available to cover all the complex underwater terrainand establish a node model for the waters to be tested.Initialize the content of Restrictions 3 and establish the constant $\mathcal{P}\left(\mathcal{S}_{i} \mid \mathbb{R}^{3}, \mathcal{A} \rightarrow 1\right)$ constant established. 


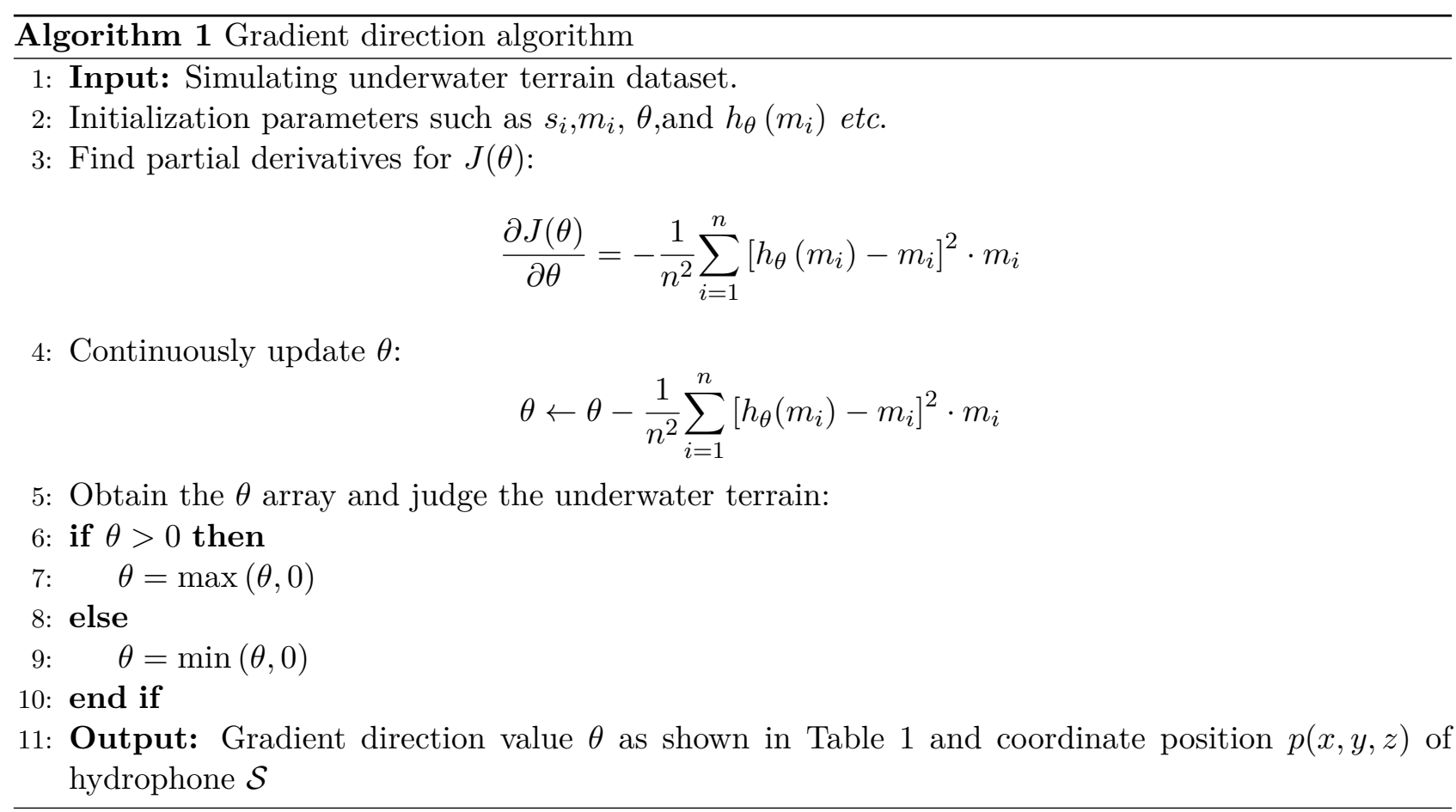

Table 1: Partial Underwater Environment Data Model Based on Gradient Direction Algorithms.

\begin{tabular}{|c|c|c|c|c|c|c|c|c|c|}
\hline Jo. & $m_{1}$ & $m_{2}$ & $m_{3}$ & $m_{4}$ & $m_{5}$ & $m_{6}$ & $m_{7}$ & $m_{8}$ & $\theta$ \\
\hline 1 & 0.02 & & & 0.0207 & 0.0954 & & & .1601 & 0.310 \\
\hline 2 & 453 & & & & & & & & \\
\hline 3 & & & & & & & & & \\
\hline 4 & 01 & & & & & & & & .0598 \\
\hline 5 & 762 & & & & & & & & -0.3564 \\
\hline 6 & 286 & 0453 & 0277 & .0174 & .0384 & & .1201 & & 0.2105 \\
\hline 7 & 21 & & & & 1674 & 1 & & & .2083 \\
\hline 8 & & & & & & & & & 0.1465 \\
\hline 9 & 3 & & & 75 & & & & & 0.0684 \\
\hline 10 & 164 & 3 & 17 & .007 & .0187 & 71 & 56 & .0697 & 0.0962 \\
\hline 11 & & & & & & & & & .0323 \\
\hline 12 & 3 & & & & & & & & .1122 \\
\hline 13 & 0079 & 6 & & 0.0 & 0.0 & 546 & 0.0528 & 0.0958 & -0.1009 \\
\hline 14 & & & & & & & & & .0955 \\
\hline 15 & & & & & & & & & .1052 \\
\hline 16 & 298 & & & 0.0 & & & & & 0.1459 \\
\hline 17 & & & & & & & & & 0.1466 \\
\hline 18 & & & & 74 & & & & & 0.1037 \\
\hline 19 & & & & & & & & & 0.0794 \\
\hline 20 & 120 & & & & 0.2565 & 0.2559 & 0.2947 & 0.411 & -0.4983 \\
\hline 21 & 473 & & & & & 0.307 & & 0.2362 & 0.383 \\
\hline 22 & & & & & & & & & 0.2353 \\
\hline 23 & 0099 & & & 0.0297 & 0.0652 & 0.1077 & 0.2363 & 0.2385 & 0.0075 \\
\hline 24 & 0115 & & & 0.0076 & 0.02 & & 0.1023 & 0.044 & -0.0931 \\
\hline 25 & & & & & & & & 0.0315 & 0.0736 \\
\hline 26 & 201 & & & 62 & & 0.0 & 541 & 21 & 0.0505 \\
\hline 27 & & & & & 0.1163 & & & 0.1119 & 0.0889 \\
\hline 28 & & & & & & & & 0.0633 & .0624 \\
\hline 29 & & & & & & & 14 & 0.0651 & 0.1896 \\
\hline 30 & 018 & & 0.0197 & 0.0622 & 0.008 & & & 0.1451 & -0.1789 \\
\hline
\end{tabular}



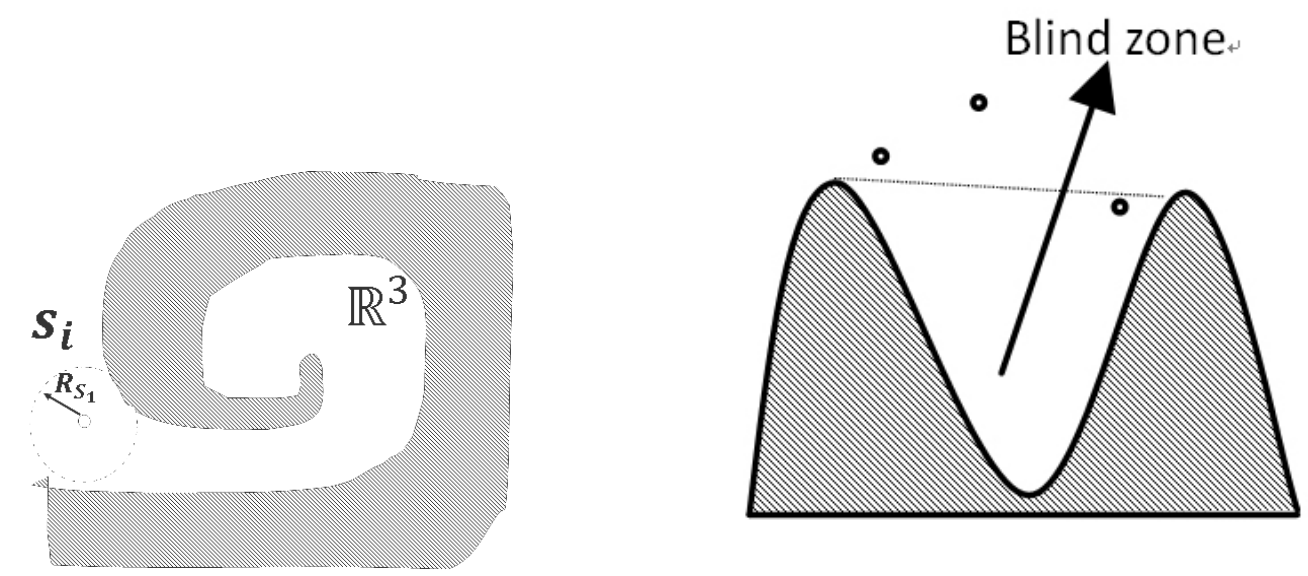

(a) Under such terrain conditions, the sensor is (b) In the case of random deployment, the presunable to perform sensing work, and even if it ence of blind spots in the hydrophone nodes is a can work, information communication cannot be high probability event. achieved.

Figure 3: Underwater blind zone and hydrophone unreachable zone.

Step3:Calculate the magnitude and direction of the $\theta$ value of each hydrophone node. If the direction is negative, then the hydrophone is in a recessed area. Room is still available for the drop, and the node position is adjusted to be in the blind spot of the hydrophone node. The node is automatically added, and the adjusted node set is output as $\mathcal{S}$.

\subsection{Experimental setup for sensor deployment}

This paper uses MATLAB to simulate the gradient direction algorithm. Mainly consider the indicators that maximize the coverage of underwater terrain and maximize the sensing data.According to the Restrictions 3,the simulation parameters in the experiment are set as follows: the monitored water area is 2000 meter $\times 2000$ meter, and the depth is 4000 meter; the underwater sensor is mainly hydrophone.Set the maximum sensing radius $R_{s_{i}}=100$ meter, the minimum sensing radius $R_{s_{i}}=$ 10 meter, the operating frequency is $35 \mathrm{kHz}$, and the initial energy is $20 \mathrm{Joule}$. The average of the results was obtained by multiple calculations, and the random deployment results were used as a reference for the algorithm during the experiment.

The advantages of the algorithm can be directly deduced from Fig.4(a), and the hydrophone node in the depressed terrain can be significantly increased, thereby increasing the probability of node deployment in the terrain region. The coverage of the number of nodes distributed under the depressed terrain is calculated and presented in Fig.4(b). Fig.4(c) shows that calculating the gradient direction results in node deployment that is more distributed in the complex space compared with that of randomly deployed nodes. Although the gradient direction algorithm can ensure that the hydrophone nodes are deployed with a high probability in the hydrophone blind spots, the calculation result is based on the following assumptions:

- The underwater topographical coordinates have been obtained by underwater sonar detection;

- Each hydrophone node can calculate its position and judge the value of $\theta$;

- Each hydrophone node can overlap with the sensing area of the adjacent node, which does not interfere with the detection;

According to the gradient descent algorithm, Section 3 describes the ability to deploy hydrophone nodes in a complex underwater environment. This ability can quickly form an underwater sensor network to calculate the sensor set with the largest coverage. According to greedy algorithm theory, the hydrophone coverage set $\mathcal{S}$ obtained in the gradient direction algorithm is not necessarily the 


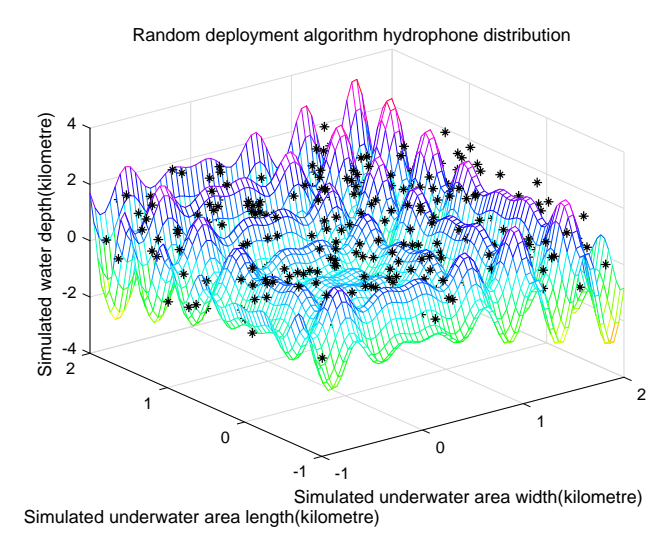

(a) Random deployment algorithm hydrophone distribution.

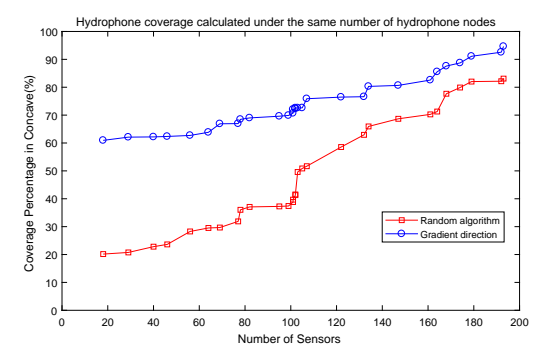

(c) Hydrophone coverage calculated under (d) the same number of hydrophone nodes.

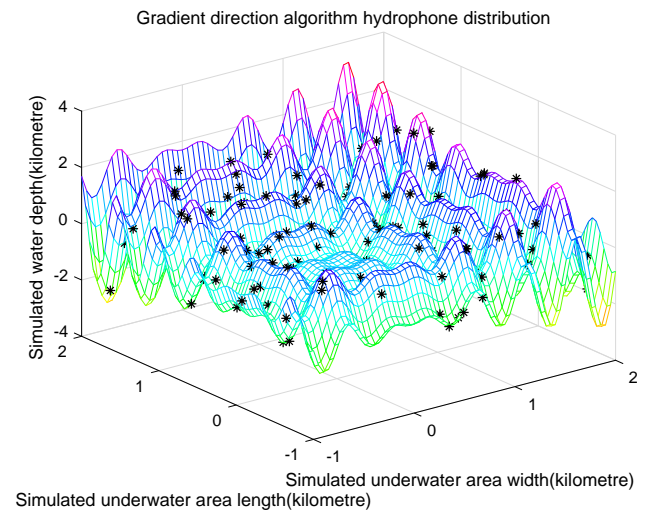

(b) Gradient direction algorithm hydrophone distribution.

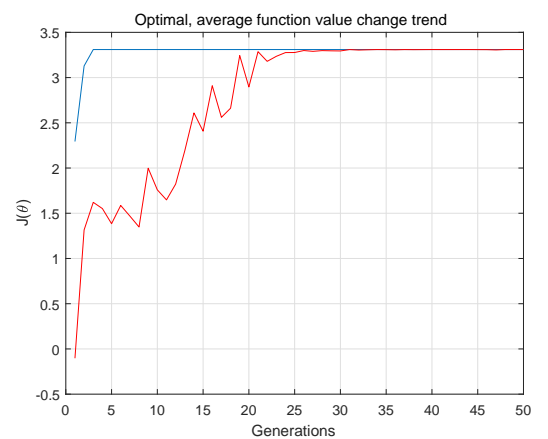

(d) Optimization and average of $J(\theta)$ after 50 iterations.

Figure 4: Hydrophone placed in a complex underwater environment.

optimal solution in the complex underwater environment.Fig.4(d) shows that Algorithm 1 optimizes the $J(\theta)$ process, from lower to higher numerical changes after 50 iterations.

According to greedy algorithm theory, each hydrophone node can calculate the regional optimization solution belonging to the local node, but all hydrophone sets are not necessarily the optimal coverage set $\mathcal{S}^{*}$. The locally optimal results calculated by each hydrophone node do not affect the local optimum calculated by subsequent sensor nodes.

\section{Calculate the approximate optimal hydrophone node coverage set}

An underwater sensor network that relies entirely on the node random deployment strategy cannot meet actual application requirements regardless of its performance and cost. The hydrological device adopts a completely random deployment strategy, which cannot guarantee network coverage and connectivity efficiency. In Section 3, the Problem 1 of maximizing the coverage of nodes in complex areas was well resolved.However, if the data sampling is poor (e.g., it contains too few sampled data and/or has an incorrect sampling), the optimal coverage has measure 100\%, but the underwater data sampling quality is not high. In addition, the excess nodes (it obtained by Algorithm 1) will increase the access collision rate of the acoustic channel and reduce the quality of network communication. This section will be based on the above coverage Algorithm 1 through the finite VC-dimension, which Brönnimann and Goodrich proposed to find the approximate optimal coverage set of the hydrophone collection system and calculate the suboptimal solution of the coverage set [13].

The main result of [4],[1] is that for hydrophone set systems with finite VC-dimension $d$, the hypothesis set hitting set $\mathscr{H}$ of size $c$ can be calculated in polynomial time $O(d c \bullet \log (d c))$. To apply this result, we must first introduce $\mathscr{H}$ and convert the hydrophone set problem into an instance of the $\mathscr{H}$ problem by calculating $\sum$.Then, we define a set system of finite VC-dimensions and use a "dual" coverage set with finite VC-dimension $d$. 


\subsection{Finite Vapnik-Červonenkis-dimension and hitting set $\mathscr{H}$}

Let $\sum=\{X, \mathcal{S}\}$ be the collection system,See Fig.5(a). The "dual" set system is defined by $\sum^{d}=$ $\left\{X^{d}, \mathcal{S}^{d}\right\}$ is defined by $X^{d}=\mathcal{S}$ and $\mathcal{S}^{d}=\left\{\mathcal{S}_{x} \mid x \in X\right\}$, where $S_{x}$ consists of all sets $S \in \mathcal{S}$ covering the complex underwater region set $x$ [1]. The "dual" set system is shown in Fig.5(b). Notice that the set of hydrophone set $\mathcal{S}$ candidates now becomes the ground set $X^{d}$.

The hitting set for $\sum^{d}=\left\{X^{d}, \mathcal{S}^{d}\right\}$ is a set $\mathscr{H}^{d} \subseteq X^{d}$ such that $\mathscr{H}^{d} \cap S^{d} \neq \emptyset$ for each set $S^{d}$ in $\mathcal{S}^{d}$ (i.e., $\mathscr{H}^{d}$ contains members from all sets of $\mathcal{S}^{d}$ ). Finding the best coverage set problem for $\sum$ is equivalent to finding the problem of smallest hitting set of $\sum$, letting $c$ be the size of the minimum hit set (the size of the minimum set cover) $[9]$.
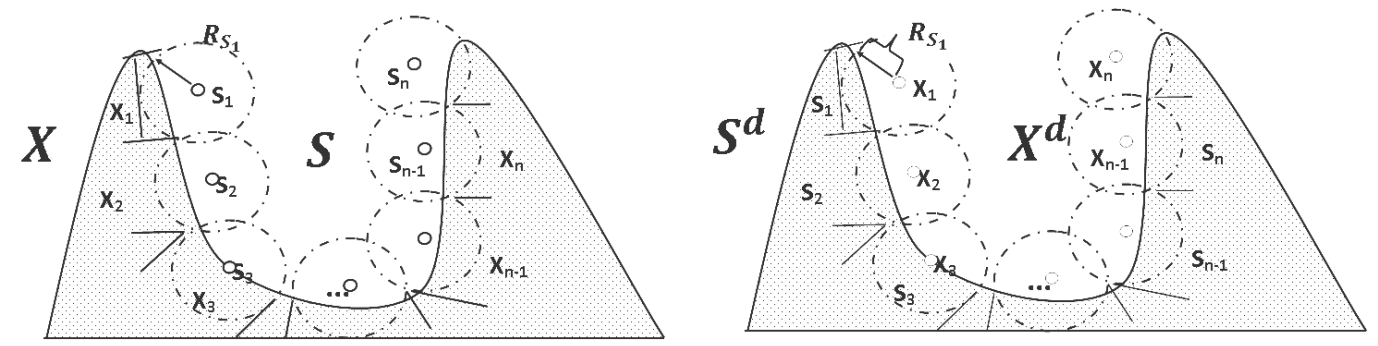

(a) $X$ represents a set of areas that need to be (b) The "dual" configuration specific subset $A \subseteq$ covered in a complex underwater environment. $\mathcal{S} X^{d}$ creation subset and region arrangement shown covers a set of hydrophone nodes in a complex in (a). The hydrophone cover set is grouped ununderwater environment. Each hydrophone node der the mark $X^{d}$, and each set $S \subseteq \mathcal{S}$ is a set sensing interval is projected onto the boundary of that covers the underwater complex terrain in the a complex underwater environment to form set $X$. boundary decomposition.

Figure 5: finite VC-dimension and a "dual" coverage set with finite VC-dimension $d$.

Definition 4. (Finite Vapnik- ̌Cervonenkis Dimension):Let $\sum^{d}=\left\{X^{d}, \mathcal{S}^{d}\right\}$ denote a set system.A set $A \subseteq X^{d}$ is said to be shattered by $\mathcal{S}^{d}$,If for any subset $B \subseteq A$ there exists some $S \in \mathcal{S}^{d}$ such that $B=A \cap \mathcal{S}^{d}$, then the set $A \subseteq X$ is shattered by $S$. The finite $V C$-dimension of $\sum^{d}$ is the cardinality of the largest shattered subset of $X^{d}$.

In other words, $A$ is shattered if each of its subsets can be induced by intersecting $A$ with some set in $\mathcal{S}^{d}$. Although it may not be possible to shatter all sets of size $d$, as long as there exists one such set we say that the VC-dimension is at least $d$. To state that a set system has VC-dimension $d$ we must prove that no set of size larger than $d$ can be shattered.

The VC-dimension of our "dual" set system $\sum^{d}=\left\{X^{d}, \mathcal{S}^{d}\right\}$ is upper bounded by the following lemma [4]:

Lemma 1. (VC-dimension for Sampled Data) The VC-dimension of the "dual" of the set system representing the sampled instance of Problem 1 is bounded by $O \log (n)$, where $n$ is the size of complex terrain data, See Definition 2.

Proof 5. (Proof of Lemma1) The coverage area under $\mathcal{M}$ for each underwater complex environment consists of up to $k=n-1$ parts. Thus, $X^{d}$ can be thought of as an arrangement of $k$-intervals, which are sets composed of at most $k$ disjointed regular intervals. Select $A \subseteq X^{d}$, where $d=|A|$. The set $A$ defines a sub-arrangement of at most $2 k d$ subdivisions in $\mathcal{M}$, and any two members of $\mathcal{S}^{d}$ within the same subdivisions induce the same subset of $A$ (i.e., $S_{i}^{d} \cap A=S_{j}^{d} \cap A$, If $S_{i}^{d}$ and $S_{j}^{d}$ are in the same subdivisions). To shatter $A$ each of its subsets must be induced, but no $S_{i}^{d}$ can induce more than one subset of A.Therefore, to induce all subsets in $A$ there must be at least $2^{d}$ subdivisions in the sub-arrangement. It is impossible to shatter $A$ if:

$$
2 k d<2^{d} \Leftrightarrow 2 k<\frac{2^{d}}{d}
$$

For $d>4, \log (2 k)<d / 2 \Rightarrow 2 k<2^{d} / d$. This observation and the conditional limit of $k=n-1$, mean that the upper limit of the finite VC-dimension is $2 \log (2 n)$. 


\subsection{Find the suboptimal sensor coverage set}

The algorithm proposed by Brönnimann and Goodrich is based on finding an $\varepsilon$-net that that approximates the optimal hitting set $\mathscr{H}[22]$. Let $\sum=\{X, \mathcal{S}\}$ denote a set system. A set $\mathscr{H} \subseteq X$ is said to be an $\varepsilon$ - net if it intersects each set $S \in \mathcal{S}$ of cardinality larger than $\varepsilon|X|$ of $\sum$. We can generalize this defnition by including an additive weight function $\omega$ on every subset of $X$. In the generalized case, an $\varepsilon-n e t$ is required to hit every $S$ with weight at least $\varepsilon \omega(X)$.

Let a net - finder of size $t$ be an algorithm $Z$ that given a weight function $\omega$ and work on set $X$,returns a $(1 / r)$ - net of size $t(r)$ for a set system $\sum$. Also,a verifier is an algorithm $Y$ that given a subset $\mathscr{H} \subseteq X$ either confirms that $\mathscr{H}$ is hitting set or returns to the hydrophone coverage set $S \in \mathcal{S}$ such that $S \cap \mathscr{H}=\emptyset$ when $\mathscr{H}$ is not the hitting set of $X$.

The main result of [6] is that given the algorithms $Z$ and $Y$, we can find the hitting set of size $t(2 c)$ (where $c$ is the optimal size) at most by executing the following algorithm: The termination

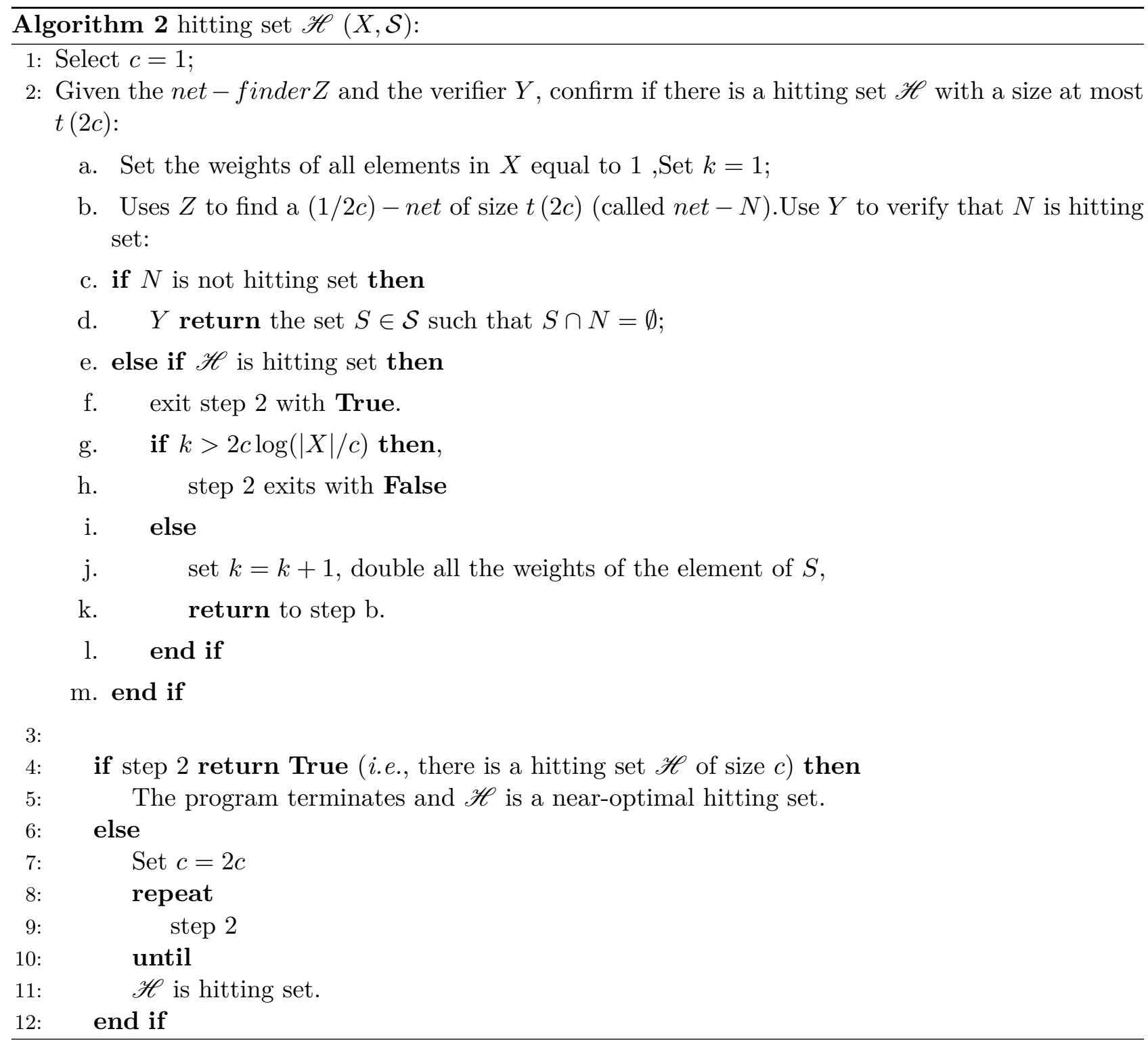

condition in step b is a remarkable result from [6]. Indeed, you can prove that step 2 always returns a hitting set within $O(2 c \log (|X| / c))$ iterations if it exists. Since step 2 returns a set of size $t(2 c)$, and because of the double condition in step 3 , the size of hitting set $\mathscr{H}$ is, at most, $t(2 c)$. The overall cost of this algorithm is $O(c \log (|X| / c))\left(T_{X}(|X|,|\mathcal{S}|, c)+T_{Y}(|X|,|\mathcal{S}|, c)\right)$ where $T_{Z}$ and $T_{Y}$ are the running times of the net finder and validation algorithms, respectively.

Under the above algorithm 2, hitting set $\mathscr{H}$ of size $c$ can be returned within the polynomial time $O(d c \bullet \log (d c))$, (i.e., the hydrophone suboptimal coverage set). We now only need to verify that 
the suboptimal solution calculated using the finite VC-dimension algorithm can achieve the maximum underwater coverage $\mathcal{S}^{*}$. In the next section, an analysis of the overall performance will be given.

\subsection{Performance analysis of optimal coverage set}

When studying the characteristics of complex environmental models, combined with the characteristics of network coverage performance and application requirements, the following three indicators are mainly considered:

- Effective coverage: The ratio between the area covered by the nodes in the network and the total size of the target area is defined as the effective coverage. To accurately analyze the effective coverage, it is necessary to calculate the area of the coverage;

- Network lifetime:The period from the start of the network to the death of the first node in the network is called the network lifetime, and its unit is the number of rounds.Fig.6(b) shows the relationship between the network lifetime and the remaining nodes of the Algorithm 1,2;

- Network energy consumption: The remaining energy of the network reflects the energy consumption of the whole network;Fig.6(c) shows the residual energy of the nodes in the Algorithm 1,2 survival on the network energy consumption during the period.

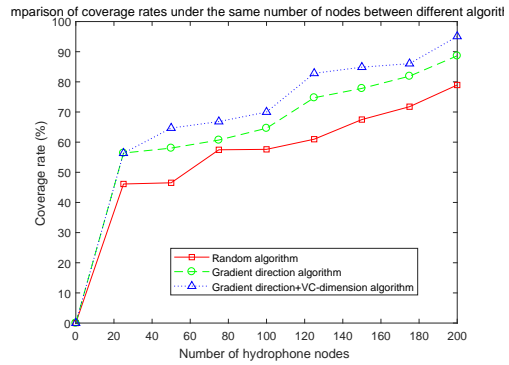

(a) Comparison of coverage rates (b) Network survival time varies (c) under the same number of nodes be- with hydrophone. tween different algorithms.

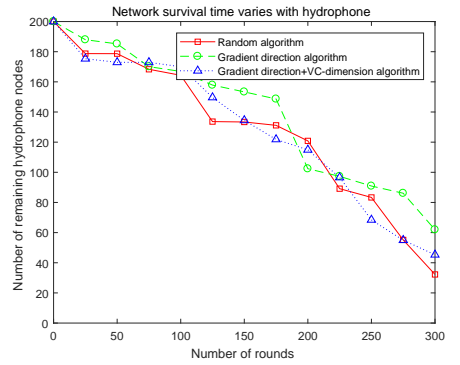
hydrophone in the UWSNs changes with time.

Figure 6: Network performance analysis of random deployment algorithm, gradient direction algorithm and VC-dimension optimization.

See Section 3.2 for the experimental environment and parameters.Fig.6 shows the relationship between the number of hydrophone nodes and the network coverage. Note that with the same number of hydrophone nodes, different coverage rates can be obtained from different algorithms. The random algorithm can be used to measure a coverage rate of approximately $70 \%$ with the same number of nodes. The gradient vector algorithm can increase the coverage of hydrophone nodes by more than 95\%, which mainly occurs after random deployment see Fig.6(a). The hydrophone node is adjusted to the blind zone of the complex sensor node caused by random deployment; therefore, without increasing the number of hydrophone nodes, sensor coverage in complex areas is effectively improved [2] . However, in this process, a large amount of hydrophone node redundancy will occur, which not only wastes resources but also places hydrophone nodes excessively, which affects the working efficiency of the nodes [29]. The combination of finite VC-dimension and gradient direction optimizes the hydrophone coverage set, and the coverage rate can reach $95 \%$, which effectively reduces the number of hydrophones. Although the finite VC-dimension algorithm is only an approximate optimal coverage set, sensor coverage has been significantly improved compared to the random deployment algorithm.

\section{Conclusion}

This paper presents a novel approach to reduce the number of hydrophone during the construction of a complex underwater sensor network model. Specifically, we incorporated sensor limitations into 
a practical sensor placement algorithm. This algorithm is based on a Gradient direction strategy that transforms the sensor placement problem into an instance of the set cover problem.

However, the calculation process revealed that the gradient direction algorithm only considers the local optimal problem, which results in a hydrophone coverage set generated by the calculation that is too concentrated in the sensor blind zone, resulting in node deployment redundancy. The hydrophone coverage set is optimized by the finite VC-dimension algorithm.

UWSNs deployment focuses not only on the geometric relationship between the physical location and spatial location of the node but also has a direct impact on the network topology, perceived data quality, and network robustness. Of course, the study of complex underwater sensor deployment issues should consider the aspects of coverage, connectivity and energy consumption, and good network connectivity ensures that the accuracy of the information collected by the underwater sensor nodes is delivered to the user terminal in a timely manner. Deployment issues also depend on support technologies, such as time synchronization and node location technology. An interesting topic for future research is to study placement strategies without underwater terrain data.Due to the limitations of the content of the article, we will focus on this in future research.

\section{Funding}

This work was supported in part by the National Natural Science Foundation of China under Grant 61962052 and Grant 61902273, in part by the Innovation Team Foundation of Qinghai Offifice of Science and Technology under Grant 2020-ZJ-903, in part by the Key Laboratory of IoT of Qinghai under Grant 2020-ZJ-Y16, in part by the Hebei IoT Monitoring Center under Grant 3142016020.

\section{Author contributions. Conflict of interest}

The authors contributed equally to this work. The authors declare no conflict of interest.

\section{References}

[1] Bartlett, P.L.; Harvey,N.(2019). Nearly-tight vc-dimension and pseudodimension bounds for piecewise linear neural networks. Journal of Machine Learning Research, 20(63), 1-17, 2019.

[2] Binh, H.T.; Hanh, N.T.(2018). Improved cuckoo search and chaotic flower pollination optimization algorithm for maximizing area coverage in wireless sensor networks. Neural computing and applications, 30(7), 2305-2317, 2018.

[3] Biswas, S.; Das, R.; Chatterjee, P.(2018). Energy-efficient connected target coverage in multi-hop wireless sensor networks. Industry interactive innovations in science, engineering and technology, Springer, 411-421, 2018.

[4] Brönnimann, H.; Goodrich, M.T.(1995). Almost optimal set covers in finite vc-dimension. Discrete \& Computational Geometry, 14(4), 463-479, 1995.

[5] Bryner, D.; Huffer, F.; Srivastava, A.; Tucker, J.D.(2016) Underwater minefield detection in clutter data using spatial point-process models. IEEE Journal of Oceanic Engineering, 41(3), 670-681, 2016.

[6] Chan, T.M.(2018). Improved deterministic algorithms for linear programming in low dimensions. ACM Transactions on Algorithms (TALG), 14(3), 30, 2018.

[7] Commuri, S.; Watfa, M.K.(2006). Coverage strategies in wireless sensor networks. International Journal of Distributed Sensor Networks, 2(4), 333-353, 2006.

[8] Darabkh ,K.A; Alsaraireh, N.R.(2018). A yet efficient target tracking algorithm in wireless sensor networks. 2018 15th International Multi-Conference on Systems, Signals \& Devices (SSD), IEEE, 7-11, 2018. 
[9] Ducoffe, G.; Habib, M.; Viennot,L.(2019). Diameter computation on $h$-minor free graphs and graphs of bounded (distance) vc-dimension. arXiv preprint arXiv:1907.04385, 2019.

[10] Harizan, S.; Kuila, P.(2019). Coverage and connectivity aware energy efficient scheduling in target based wireless sensor networks: an improved genetic algorithm based approach. Wireless Networks, 25(4), 1995-2011, 2019.

[11] Hu, W.; Yao, W.H.; Hu, Y.W.; Li, H.H.(2019). Selection of cluster heads for wireless sensor network in ubiquitous power internet of things. International Journal of Computers Communications \& Control, 14(3), 44-358, 2019.

[12] Jagtap, A.M.; Gomathi, N.(2020) Energy efficient sensor deployment with tcov and ncon in wireless sensor networks: Energy efficient sensor deployment with tcov. International Journal of Embedded and Real-Time Communication Systems (IJERTCS), 11(1), 1-22, 2020.

[13] Kjos-Hanssen, B.; Felix, C.J.; Kim, S.Y.; Lamb, E.; Takahashi, D.(2020). Vc-dimensions of nondeterministic finite automata for words of equal length. arXiv preprint arXiv:2001.02309, 2020.

[14] Lacharité, M.; Brown, C.J.; Gazzola, V.(2018). Multisource multibeam backscatter data: developing a strategy for the production of benthic habitat maps using semi-automated seafloor classification methods. Marine Geophysical Research, 39(1-2), 307-322, 2018.

[15] Luo, X.B.; Xu, D.M.; Hu, J.J.; Hu, M.(2014). Application research of 3d imaging sonar system in salvage process. Applied Mechanics and Materials, Trans Tech Publ, 643, 279-282, 2014.

[16] Morozs, N.; Mitchell, P.D.; Zakharov, Y.; Mourya, R.(2018). Robust tda-mac for practical underwater sensor network deployment: Lessons from usmart sea trials. Proceedings of the Thirteenth ACM International Conference on Underwater Networks \& Systems, ACM, 11, 2018.

[17] Mridula, K.M.; Ameer, P.M.(2018). Three-dimensional sensor network connectivity considering border effects and channel randomness with application to underwater networks. IET Communications, 12(8), 94-1002, 2018.

[18] Nazarzehi, V.; Savkin, A.V.(2018). Distributed self-deployment of mobile wireless 3d robotic sensor networks for complete sensing coverage and forming specific shapes. Robotica, 36(1), 1-18, 2018.

[19] Nielsen, P.L.; Muzi, L.; Siderius, M.(2017). Seabed characterization from ambient noise using short arrays and autonomous vehicles. IEEE Journal of Oceanic Engineering, 42(4), 1094-1101, 2017.

[20] Ojha, T.; Misra, S.; Raghuwanshi, S.(2015). Wireless sensor networks for agriculture: The stateof-the-art in practice and future challenges. Computers and Electronics in Agriculture, 118, 66-84, 2015 .

[21] Osamy, W.; Khedr, A.M.(2018). An algorithm for enhancing coverage and network lifetime in cluster-based wireless sensor networks. International Journal of Communication Networks and Information Security, 10(1), 1-9, 2018.

[22] Pinto, L.; Gopalan, D.(2019). Limiting network size within finite bounds for optimization. arXiv preprint arXiv:1903.02809, 2019.

[23] Poduri, S.; Pattem, S.; Krishnamachari, B.; Sukhatme, G.(2006). Sensor network configuration and the curse of dimensionality. Proc. Third Workshop on Embedded Networked Sensors (EmNets 2006), Cambridge, MA, USA. Citeseer, 2006.

[24] Saffran, J.R.; Kirkham, N.Z.(2018). Infant statistical learning. Annual review of psychology, 69, 2018. 
[25] Saikia, M.; Hussain, M.A.(2019). Wireless sensor node deployment strategy for hilly terrains-a surface approximation based approach. IET Wireless Sensor Systems, 9(5), 284-294, 2019.

[26] Shakkottai, S.; Srikant, R.; Shroff, N.B.(2005). Unreliable sensor grids: Coverage, connectivity and diameter. Ad Hoc Networks, 3(6), 702-716, 2005.

[27] Tam, N.T.; Hai, D.T.(2018). Improving lifetime and network connections of 3d wireless sensor networks based on fuzzy clustering and particle swarm optimization. Wireless Networks, 24(5), 1477-1490, 2018.

[28] Yao, L.; Du, X.J.(2020). Identification of underwater targets based on sparse representation. IEEE Access, 8, 215-228, 2020.

[29] Zhang, C.L.; Bai,X.L.; Teng,J.(2010). Constructing low-connectivity and full-coverage three dimensional sensor networks. IEEE Journal on Selected Areas in Communications, 28(7), 984-993, 2010 .

[30] Zheng, X.; Feng, C.; Li, T.Y.(2019). Analysis of autonomous underwater vehicle (auv) navigational states based on complex networks. Ocean Engineering, 187, 106141, 2019.

[31] Zou, Y.; Chakrabarty, K.(2003). Sensor deployment and target localization based on virtual forces. IEEE INFOCOM 2003. Twenty-second Annual Joint Conference of the IEEE Computer and Communications Societies (IEEE Cat. No. 03CH37428), IEEE, 2, 1293-1303, 2003.

\section{(c) (i) (8)}

Copyright (C)2020 by the authors. Licensee Agora University, Oradea, Romania.

This is an open access article distributed under the terms and conditions of the Creative Commons Attribution-NonCommercial 4.0 International License.

Journal's webpage: http://univagora.ro/jour/index.php/ijccc/

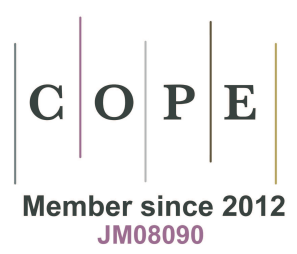

This journal is a member of, and subscribes to the principles of, the Committee on Publication Ethics (COPE).

https://publicationethics.org/members/international-journal-computers-communications-and-control

Cite this paper as:

Yao, L.; Du, X. (2020). Sensor Coverage Strategy in Underwater Wireless Sensor Networks, International Journal of Computers Communications \& Control, 15(2), 3659, 2020.

https://doi.org/10.15837/ijccc.2020.2.3659 